\title{
Enhanced Multi-layer Lens-Let Array for Extreme Angle Solar Collection
}

\author{
Rakan E. Alsaigh, ${ }^{1}$ Ralf Bauer, ${ }^{2}$ and Martin P.J. Lavery ${ }^{1}$ \\ 'School of Engineering, University of Glasgow, Glasgow, UK \\ Department of Electronic and Electrical Engineering, University of Strathclyde, Glasgow, UK \\ Authors e-mail address: r.alsaigh.1@research.gla.ac.uk,ralf.bauer@strath.ac.uk and martin.lavery@glasgow.ac.uk
}

\begin{abstract}
Deployment of solar panels on the side of buildings leads to very-low collectionefficiency. We present an enhanced multi-layer lens-let array that increases the daily generated power at near vertical deployment by a factor of 4.783 .
\end{abstract}

C 2019 R. E. Alsaigh, R. Bauer, and M. P.J. Lavery

OCIS codes: $350.0350,350.6050$. For codes, see http://www.osapublishing.org/submit/ocis/.

Solar photovoltaics (PV) are playing a central role in meeting the global and future energy demand [1]. However, the transition towards a sustainable future where efficient solar PV is expected to play a central role. Traditionally, solar technologies have a limited field of view, which prevent efficient deployment of solar PV in mobile platforms, sustainable communication systems and autonomous vehicles [2,3], as mechanical tracking is required. Mechanical trackers have been conventionally used to provide solar panels with a normal incident towards the sun, however, they are only suitable for open-spaces and are expensive to build and maintain. A further opportunity is the deployment of PV onto the side of buildings [4], where the extreme angle of incidence with respect to the sun severally limits optical performance. Wide field of view novel optics are a strong candidate to provide wider solar collection capabilities for solar power systems and increase their overall acceptance angles [5,6]. Alsaigh et. al. previously developed a proof of principle novel multi-layer lens-let array (MLA) that increased the effective field of view of collection for the solar panel that potentially could remove the requirement of mechanical tracking and offer the opportunity for near vertical deployment of solar PV [5,7].

We will present our latest achievements in developing a novel multi-layer lens-let array design that provides ultrawide field of view of \pm 80 degrees for efficient solar collection from a wide range of input angles [5,7]. Our design incorporates two small-aperture hemispheric lens-let arrays bonded together by their curved faces to form a telecentric arrangement that has field of view (FOV) of 80 degrees [7,8]. This arrangement allows the efficient capture of input rays incident into the top array by leading them through the opposite lens or redirecting them through one of its neighbouring lenses in the second array, see Fig. 1(a). We prototyped this design using a stereolithographic 3Dprinting technology, see Fig. 1(b), and integrated it into an off-the-shelf Amorphous Silicon solar panel (Sanyo, AM1815CA), which experimentally demonstrated an increased multiplicative power factor of 1.038 to 4.783 over the flat counterpart, depending on the panel's latitudinal tilt angle as shown in Fig. 2(a). Additionally, a ray tracing model of our design was developed to compute the optical power collected at the solar panel surface and the angle at which the rays input the solar panel pane. These models predict that the majority of optical power is coupled into five neighbouring lens-lets on the second layer of the element, at angles that match the efficient acceptance window of the solar panel.

(a)
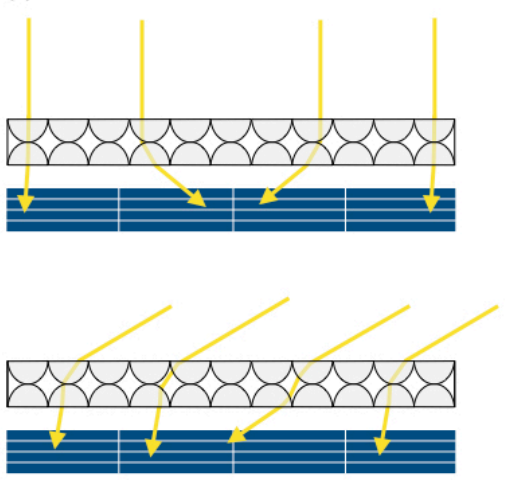

(b)

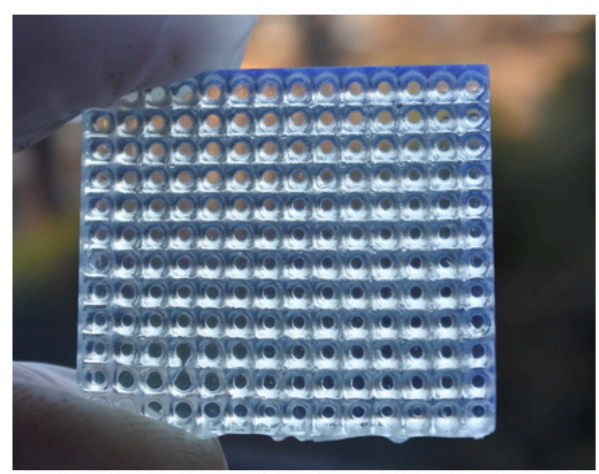

Fig. 1. Ultra-wide field of view multi-layer lens-let array. (a) Ray tracing schematic of our multi-layer lens-let arrays, with simulated rays 
entering the system at incident angle of 0 degree (top) and 60 degrees (bottom). (b) photograph of our 3D-printed optical prototype.

Based on this simulation, we will present an enhanced version of our previously reported multi-layer lens-let array with an optimized optical performance compared to the prototype design outlined in [5,7]. To achieve this enhanced design, a gradient-free finite element optimization formulation was employed to optimize the distribution and dimensions of the lenses in the MLA. Moving away from linearly distributed array could increase the collected optical power considerably. The optimum position and radius of each single lens in the lower array layer was determined by maximizing the number of collected rays and minimising internal reflections, through the use of Nelder-Mead numerical algorithm. This algorithm solves for a set of objective function to return the maximum power collected at the back surface of the MLA based on a set of bounded constraints. Full system simulations indicated that the optimised design to outperform our previously reported design, see Fig 2(b), where the expected power of our MLA is increased by a multiplicative factor of 1.074 to 2.132 . With further development our optimization algorithm can be used to develop a various use-cases design that is suitable for a wide range of applications with no fixed alignment towards the sun such as drones, or case-specific design for applications that optimally operate at a specific incident angle such as building exteriors.

(a)

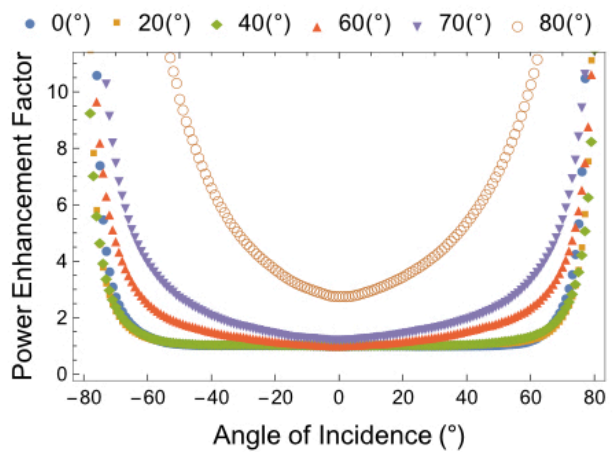

(b)

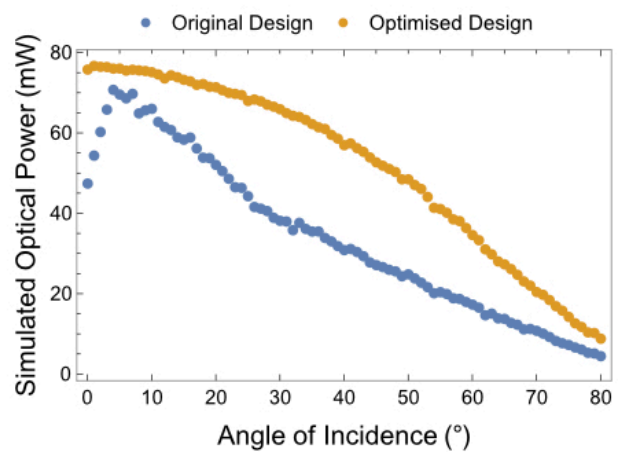

Fig. 2. Experimental and simulated results. (a) Multiplicative gain factors for the power produced by element-integrated PV panel over its flat counterpart at longitudinal tilt angle of $0^{\circ}, 20^{\circ}, 40^{\circ}, 60^{\circ}, 70^{\circ}$ and $80^{\circ}$. (b) Simulated optical power for rays entering a single central lens and collected at the back surface of the second array for the original design (blue) and an optimized design (yellow)

We will present the experimental results and characterizations, including measured open circuit voltage, short circuit current density and power gains achieved through the integration of our optical prototype into an off-the-shelf solar panel. We will discuss our approach in designing, fabricating and post-processing our 3D-printed multi-layer lens-let array. Our simulation results will also be presented to show the predicted angular deflection of the rays and the total optical power outputting our system. Based on this simulation, we will discuss our optimization approach and results to enhance our linear array design into a more compact distribution of lens-lets. Our enhanced multi-layer lens-let array could be tailored to provide an efficient route to power fixed or mobile systems and enables the effective use of electric vehicles, aircrafts and drones in future applications.

\section{References}

[1] Steven Chu, Yi Cui and Nian Liu, "The path towards sustainable energy," Nature Materials 16, 16-22 (2017).

[2]. Martin P. J. Lavery, Mojtaba Mansour Abadi, Ralf Bauer, Gilberto Brambilla, Ling Cheng, Mitchell A. Cox, Angela Dudley, Andrew D. Ellis, Nicolas K. Fontaine, Anthony E. Kelly, Christoph Marquardt, Selaelo Matlhane, Bienvenu Ndagano, Francesco Petruccione, Radan Slavík, Filippo Romanato, Carmelo Rosales-Guzmán, Filippus S. Roux, Kobus Roux, Jian Wang and Andrew Forbes, “Tackling Africa’s digital divide,” Nature Photonics 12, 249-252 (2018).

[3]. Xian-Zhong Gao, Zhong-Xi Hou, Zheng Guo, Xiao-Qian Chen, "Reviews of methods to extract and store energy for solar-powered aircraft," Renewable and Sustainable Energy Reviews 44, 96-108 (2015).

[4]. Akash Kumar Shukla, K. Sudhakar, Prashant Baredar, "A comprehensive review on design of building integrated photovoltaic system," Energy and Buildings 128, 99-110 (2016).

[5]. Rakan E. Alsaigh, Ralf Bauer and Martin P.J. Lavery, "Efficient solar collection beyond the etendue limit," arXiv:1808.06973 [physics.appph], (2018).

[6]. K. Shanks, S. Senthilarasu, T. K. Mallick, "Optics for concentrating photovoltaics: Trends, limits and opportunities for materials and design,” Renew. Sus. Energ. Rev. 60, 394-407 (2016).

[7]. Rakan E. Alsaigh, Ralf Bauer and Martin P.J. Lavery, "Ultra-wide field of view lens-let array for efficient solar collection," Conference on Lasers and Electro-Optics (CLEO 2019), San Jose, California, USA, May 2019.

[8]. M. Rezaei, I. H. Nia, A. Bonakdar, H. Mohseni, "Simple telecentric submillimeter lens with near-diffraction-limited performance across an 80 degree field of view," App. Opt. 55, 8752- 8756 (2016). 